\title{
A realization method of multi-protocol label switching traffic engineering based on logical hierarchy
}

\author{
$\operatorname{Jia} \operatorname{Ren} *^{1, \text { a }}$ \\ ${ }^{1}$ Xi'an fanyi university, Xi'an, China \\ Shuhua_y@126.com
}

Keywords: Hierarchical, Multi-protocol Label Switching, Traffic Engineering, LSR

\begin{abstract}
Aiming at the shortcomings of traditional single-level methods and existing multi-level network traffic engineering (TE), a realization method of multi-protocol label switching traffic engineering (MPLS TE) based on logical hierarchy is put forward. By using TE to achieve the equilibrium distribution of network load, this study interprets the design thought, routing and forwarding services of MPLS TE based on logical hierarchy, and illustrates working process by means of examples. The scheme points out that the size of each network needs to be further researched.
\end{abstract}

\section{Introduction}

With the soaring development of network technology, users' requirement for quality of service (QoS) of networks increases continuously. However, network congestion frequently happens due to scant network resources and unevenly distributed traffic. The former can be solved by means of upgrading infrastructures in order to provide more network resources while traffic engineering (TE) is employed to adjust and control network functions for the latter so as to provide more reasonable traffic distribution. TE is a macroscopic-regulating and microscopic-controlling network engineering technology which maps the traffic flow to actual physical paths and automatically optimizes network resources simultaneously to realize services of specific application programs ${ }^{[1,2]}$.

The basic thinking of multi-protocol label switching (MPLS) is to simplify protocol stacks and achieve close combination of Layer 2 switching and Layer 3 routing so as to make a simple but effective multi-protocol technology platform for various high-level business. illustrates that MPLS can identify and measure the traffic flow between specific ingress nodes and egress nodes. It classifies packages flowing into the network at first and labels those in the same routing group ${ }^{[3,4]}$. The labels are recognized as the only mark that decides routing operation. Label switch routers (LSRs) at each network node are responsible for obtaining topology information of a region by operating interior routing protocols such as OSPF and IS-IS. Besides, they execute label distribution protocols at the same time to map the destination address and labels of packages and distribute mapping information.

The major technology of traditional MPLS TE is limited in single-level TE which is with small coverage. For example, a local area network of a school can form a single-level network to solve the traffic of users in the MPLS area, which means, that congestion will also appear if interior users visit exterior resources. As to wide area networks, MPLS networks are built inevitably based on IP to reach a certain MPLS area. When multiple users of a MPLS area need to access the same destination or resources in the MPLS area, the shortest path of IP networks is bound to be congested while other two long paths of routers in the two areas are not fully used. As the network size augments, the routing table of each router increases by folds, which not only consumes router memory but also takes more CPU time to scan the table. In such context, more bandwidth is needed to send reports of relevant status and more time to accomplish a link ${ }^{[5]}$.

Aiming at the shortcomings of traditional single-level MPLS TE, a network TE based on physical network structure is generally adopted, which is built after optimizing the network physical structure. 
But it cannot effectively amend the traditional single-level network TE with existing physical network. In addition, this physical structure shows weak multi-level MPLS expansibility as the number of users increases ${ }^{[6,7,8]}$.

This study proposes a new method of MPLS TE targeting the weakness of traditional single-level methods and existing multi-level network TE.

\section{Design thinking of MPLS TE based on logical hierarchy}

Geographical scopes, assigned LSRs and hierarchical structures of MPLS systems have to be taken into account while designing a MPLS TE system based on logical hierarchy.

Firstly, management strategies are adopted to determine the scope of the MPLS systems by dividing certain areas with similar data switching amount into one level network. When two neighboring low-level networks increase data switching amount to that close to the interior switching amounts of the network, they can be integrated into one network. As the data switching amount of two neighboring low networks elevates so enormously that significantly surpasses the interior switching amounts of the networks, the two networks should be redistricted.

Secondly, after region division and confining network levels, roles of LSRs in networks require to be identified, i.e. determine whether each LSR belongs to edge LSR or core LSR. A same LSR probably plays different routing roles in different network classes: it may be an edge LSR of low level networks or a core LSR in networks at higher level. As an edge LSR, it is responsible for routing computation of different networks at the same level as well as the LSRs in the network. For core LSRs, they are merely in charge of the forwarding and label switching of interior data packets in this network.

Thirdly, the hierarchical structure of MPLS networks is divided according to certain management strategies after confining LSRs. For examples, managing the network depends on geographical location by classifying small areas reaching conditions as low network areas, integrating several neighboring low networks into a high network area, and so on, until the whole network is distributed ${ }^{[9,}$ 10].

\section{Routing and forwarding of MPLS TE based on logical hierarchy}

As illustrated in Figure 1, there are three hierarchies in the MPLS network models based on logical hierarchy. The lowest hierarchy directly provides service for users, such as J and K nodes, of which the father node LSRA is an edge LSR of the MPLS area. Apart from sending data packets to users' nodes ( $\mathrm{J}$ and K), LSRA also builds connections with edge LSRs like LSRB, forms label switching path (LSPs) in MPLS area (area III-1) at this hierarchy and sends data packets to these nodes. When the third hierarchy is abstracted to the second hierarchy, each small area at the third hierarchy is abstracted to an equivalent LSR, abstracting area III-1 to LSR-1 for instance, leaving the links of various areas unchanged. The abstraction of second to the first hierarchy is performed in the same way. In this way, LSRB is both the edge LSR of area III-1 and the core LSR of area II-1.

LSPs are built through CR-LDP or RSVP. While building a LSP, the links within areas are established in terms of the state information of links within areas, while inter-area links are built according to inter-area state information of links, which is published by inter-area interface LSR. For example, III-1 and III-2 at the third hierarchy acquire link state information of links through LSRB and LSRC. Besides, building LSPs of the two areas dose not require to know their interior physical structures and link states.

\subsection{Link building in areas at the third hierarchy}

Suppose that node J (address: 13) expects to exchange data with node L (address: 36). Node J first sends a request to LSRA for building connections with node L, but node LSRA finds that the address 36 does not belong to its administrative scopes so it looks for relevant routing tables in the third hierarchy MPLS area. The finding shows that address 36 is in the scope of node LSRB and then 
LSRA sets up LSPs with node LSRB, i.e. LSRA-LSRB, through CR-LDP or RSVP. Afterwards, node LSRB links with node L and builds J-LSRA-LSRB-L. Finally, node J can exchange data with node $\mathrm{L}$.

\subsection{Link building in areas at the second hierarchy (with areas at the third hierarchy)}

If node $\mathrm{K}$ (address: 13 ) needs to exchange data with node $M$ (address: 78), it sends a request to LSRA to link with node $\mathrm{M}$ at first. Finding that the address 78 is out of the administrative scope, LSRA searches the routing label in the MPLS, confirming that the address 78 comes from other areas so it directly seeks the neighboring areas through the edge LSRs of the area. The request is sent by LSRB and LSRP at the same time. The second hierarchy model can be used for interpreting. LSR-2 finds that node $\mathrm{M}$ belongs to its scope (delivered by the edge LSR of area III-2) while node K is in the command of LSR-1, and sets up a LSP, i.e. K-LSR-1-LSR-2-M, through CR-LDP or RSVP. The better path of the two physical links from LSR-1 to LSR-2 is selected depending on inter-area link state information announced by the edge LSR (LSRB、LSRC、LSRP、LSRQ) of the two areas, indicated as link LSRB - LSRC. Building of area links is not directly related to the link information of their areas, i.e. area III-1 does not know interior information of area III-2 and it does not need to know as well. The process of LSRC to build links with node $M$ is as same as the former. After the above two steps, a LSP from $\mathrm{K}$ to $\mathrm{M}$ is established, i.e. $\mathrm{K}-\mathrm{LSRA}-\mathrm{LSRB}-\mathrm{LSRC}-\mathrm{LSRD}-\mathrm{M}$, and subsequently, node $\mathrm{K}$ can exchange data with node $\mathrm{M}$.

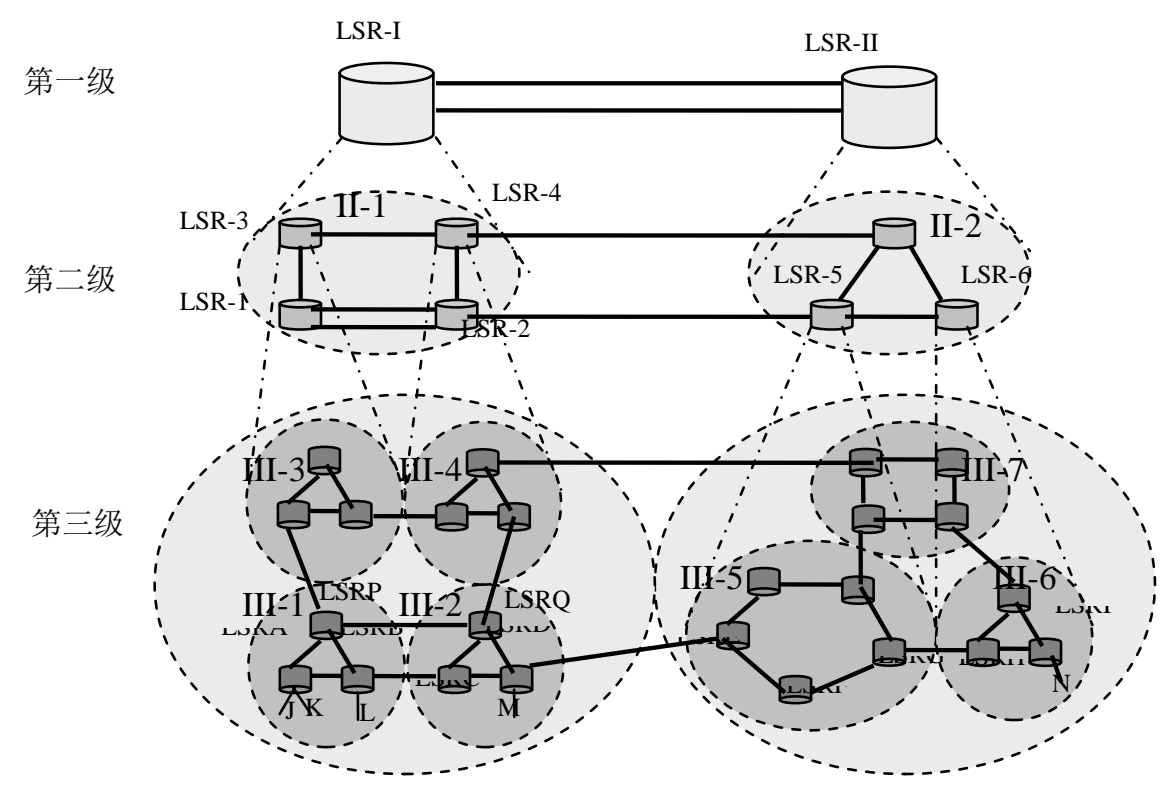

Figure 1 MPLS network models

\subsection{Link building in areas at the first hierarchy (with areas at the second hierarchy)}

Node K (address: 13) is assumed to exchange data with node $N$ (address: 102). To begin with, node $\mathrm{K}$ sends a request to LSRA for connections with node $\mathrm{K}$. When finds node $\mathrm{N}$ is out of area III-1, LSRA seeks area II-1 where node K belongs to and results show that it is not in the area as well. Next, LSRA searches areas of node $\mathrm{K}$ at higher hierarchy and finally finds that node $\mathrm{N}$ is in LSR-II. Using the same method, the first hierarchy LSP is established, i.e. LSR-I - LSR-II. One of the two physical links is selected and supposed to be LSRD - LSRE. The second hierarchy LSP is LSR-1-LSR-2, LSR-5-LSR-6, i.e. LSRB - LSRC, LSRG - LSRH, while that of the third hierarchy is LSRALSRB, LSRC-LSRD, LSRE-LSRF-LSRG, LSRH-LSRI. Through the LSP built above from $\mathrm{K}$ to $\mathrm{N}$, i.e. $\mathrm{K}$ - LSRA - LSRB - LSRC - LSRD - LSRE - LSRF - LSRG - LSRH - LSRI$\mathrm{N}$, node $\mathrm{K}$ can exchange data with node $\mathrm{N}$. 
From the analysis above, it can be seen that on the one hand logical hierarchy of networks limits the scope of broadcast domains and thereby can effectively divide broadcast domains. On the other hand, it also shortens the length of routing table of each router. The realization of MPLS TE based on logical hierarchy neither relies on network physical structure nor needs to change physical structure of previous networks. It achieves network division from logical perspective. This kind of TE based on logical hierarchy will help to promote the development of network in the future.

\section{Conclusions}

The realization of MPLS has a strategic meaning of TE. By means of integrated models, MPLS offers almost all the functions of overlapping models and creates possibility for the automatic realization of TE. Therefore, MPLS is certainly a better scheme among various realization schemes of TE. With rapid development of network, achieving TE on the basis of existing network helps enlarge network size in the future. Therefore, MPLS TP based on logical hierarchy is no doubt a better scheme. Obviously, many aspects of the scheme need to be further amended, such as LSRs. Moreover, LSRs are expected to change roles in different hierarchies in time and the size of each network hierarchy requires to be further researched in hierarchical network.

\section{Acknowledgement}

In this paper, the research was sponsored by the science research projects of education department in Shaanxi provincial government (Project No. 16JK2078).

\section{References}

[1] Sun Jie, Jiang Jie, Yang Daiyun. A method of MPLS-TP shared ring protection in PTN[J]. Optical Communication Technology, 2014,3:21-23.

[2] Zhang Shunmiao. MPLS TE and VPN implementation method based on software defined network [J]. Journal of Fuzhou University: Natural Science Edition,2015,4,43(3):340-344.

[3] Gu Weijiang, Liu Xianwen. Multidimensional awareness-based signaling mechanism in MPLS-TP-based optical networks[J]. 2014,10(5):16-18

[4] Zhou Naifu. Weighted Fair Queuing and Feedback Round Robin Scheduling Algorithm on MPLmS [J]. Bulletin of Science and Technology,2014,1,30(1): 174-178.

[5] Xiong Xiaoping, Tan Jiancheng, Lin Xiangning. Routing Algorithm for Communication System in Wide-Area Protection Based on MPLS[J]. Transactions of China Electrotechnical Society. 2013,6,28(6):257-263.

[6] He Shaotong, Xue Lunsheng, Liu Zan. Research on the De-noising of Reroute Signals in the Troposphere Scattering with MPLS[J]. Fire Control \& Command Control,2016,12(41):130-137.

[7] HELVOORT H. Draft-helvoort-mpls-tp-ring-protection-switching-02: IETF Draft[S]. New York: IETF, 2012.

[8] Requirements for Operations, Administration, and Maitenance(OAM) in MPLS Transport Networks[S]. IETF RFC5860-2010.

[9] Liu Fengqing, Jaume Comellas. Impact of Optical Hop Constraints on the IP/MPLS-over-WSON Networks CAPEX[J]. China Communications, 2015,11: 112-118.

[10] Michal Pioro, Artur Tomaszewski, Cezary Zukowski etc. Optimized IP-Based vs. Explicit Paths for One-to-One backup in MPLS Fast Reroute[J].IEEE/Networks,2010. 